\title{
Experimental investigations of the turbulent/non-turbulent interface over surface with spanwise heterogeneity
}

\author{
Yanguang Long ${ }^{1}$, Jinjun Wang ${ }^{1 *}$, Chong Pan ${ }^{1}$ \\ ${ }^{1}$ Beijing University of Aeronautics and Astronautics, Fluid Mechanics Key Laboratory of Ministry of \\ Education, Beijing, China \\ *jjwang@buaa.edu.cn
}

\begin{abstract}
The sharp but irregular interface that separates the instantaneous turbulent and irrotational flows is termed as the turbulent/non-turbulent interface (TNTI). TNTI can be widely observed in various types of flow, such as turbulent boundary layers, jets and combustion flame fronts. Due to its importance on the intermittency and entrainment process, TNTI has been widely explored in its geometry and dynamic properties (da Silva et al., 2014). Most of the studies focus on the TNTIs in smooth plane boundary layer, while few investigate the effects of wall shapes. However, the wall conditions in many engineering applications are complex and heterogeneous, which will induce large-scale heterogeneity (Barros and Christensen, 2014) and require further investigations. To shed new light on the intermittency and entrainment above complex surfaces, the TNTI over spanwise heterogeneity are investigated here with time-resolved stereoscopic PIV (TR-SPIV).

The model and TR-SPIV experimental set-up are shown in Fig. 1. The experiments are conducted in the low-speed water channel at Beijing University of Aeronautics and Astronautics. The spanwise distance $S$ between two adjacent ridges is $S /\langle\delta\rangle=1.35$, where $\langle\delta\rangle$ is the spanwise-averaged boundary layer thickness. This spanwise distance is selected to induced strong secondary vortices (Vanderwel and Ganapathisubramani, 2015; Wangsawijaya et al., 2020). The Reynolds number based on the streamwise location $x$ is $R e_{x}=7.2 \times 10^{5}$. The field of view is around $2 S \times 1.8 S$, and is captured by two CMOS cameras $(2048 \times 2048$ pixel) with sampling rate as $500 \mathrm{~Hz}$. The averaged resolution is about 8 pixels per Kolmogorov scale (calculated at $y /\langle\delta\rangle=0.6$ ), which is high enough for TNTI-related research (Borrell and Jiménez, 2016). The TNTI is detected by the magnitude of local enstrophy $\omega^{2} / 2$, and the threshold is selected to be the value where changing the threshold has the smallest influence on the TNTI-mean-height (Watanabe et al., 2018).

The time-mean velocity and TNTI location are present in Fig.2(a). A pair of counter-rotating largescale secondary vortices (SVs) are induced over the ridge-type roughness. At the position where SVs induce upwash flow, a low-momentum pathway (LMP) can be observed, while the time-mean height of TNTI $\left\langle y_{I}\right\rangle$ is brought higher. As a contrast, where downwash flow induces high-momentum pathway (HMP), $\left\langle y_{I}\right\rangle$ is lower.
\end{abstract}

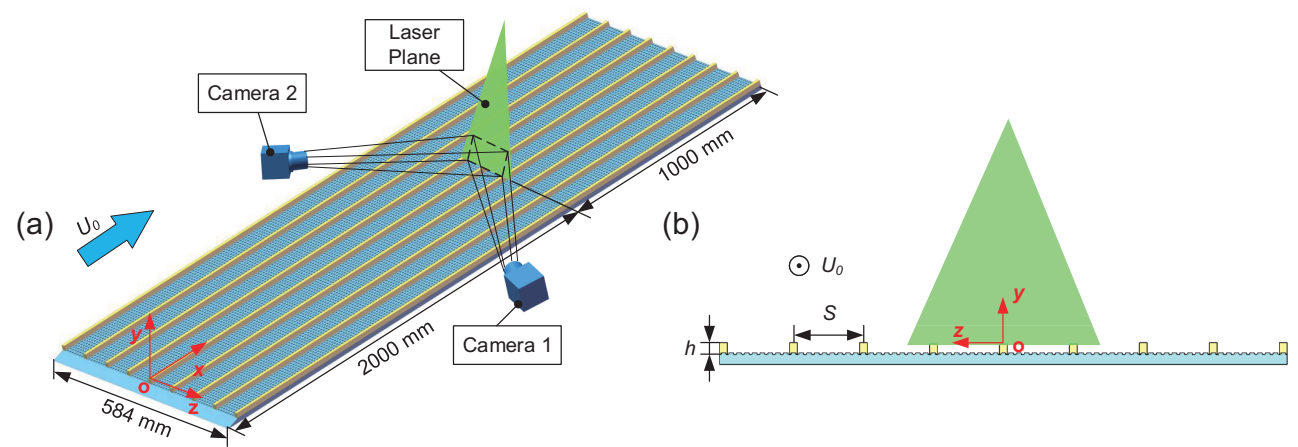

Figure 1: Model and TR-SPIV experimental set-up. (a): axis view; (b): spanwise-vertical cutaway view 

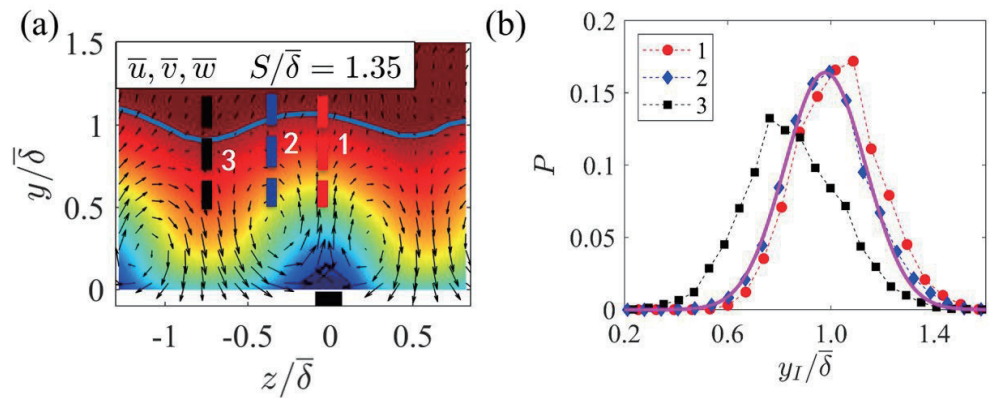

Figure 2: (a) The time-mean velocity and TNTI location. Background colormap: streamwise velocity; black vectors: spanwise and vertical velocity; blue curve: TNTI location. (b) the p.d.f. of TNTI height at three locations denoted in (a); the pink curve is the Gaussian distribution.

TNTI properties are further discussed from two aspect. The geometry properties are firstly investigated. The fractal dimension of the TNTI keeps as 2.3 along the spanwise direction. This value is consistent with the result over smooth plate (Borrell and Jiménez, 2016; Wu et al., 2020) and riblets plates(Cui et al., 2019), which indicates that the wall shapes do not influence the multiscale properties of the TNTI. The streamwise wavelength of the TNTI $\left(\lambda_{I}\right)$ is further obtained by calculating the streamwise pre-multiplied spectrum of the TNTI. It is found that at each spanwise location, $\lambda_{I}$ is identical to the wavelength of streamwise velocity fluctuation at the TNTI mean height. This shows that the large-scale fluctuation of TNTI is controlled by the large-scale streamwise velocity fluctuation structures. Secondly, the p.d.f. of TNTI instantaneous height is investigated, as shown in Fig. 2(b). It can be observed that the p.d.f. of TNTI height above LMP shows a negative skewness, while the p.d.f. above HMP skews positively. A closer look at instantaneous structures shows that the skewness is attributed to the different probability of Q2/Q4 events in LMP and HMP.

\section{Acknowledgements}

This work was supported by National Natural Science Foundation of China (grant number 91852206, 11721202)

\section{References}

Barros JM and Christensen KT (2014) Observations of turbulent secondary flows in a rough-wall boundary layer. Journal of Fluid Mechanics 748:R1

Borrell G and Jiménez J (2016) Properties of the turbulent/non-turbulent interface in boundary layers. Journal of Fluid Mechanics 801:554-596

Cui G, Pan C, Wu D, Ye Q, and Wang J (2019) Effect of drag reducing riblet surface on coherent structure in turbulent boundary layer. Chinese Journal of Aeronautics 32:2433-2442

da Silva CB, Hunt JC, Eames I, and Westerweel J (2014) Interfacial layers between regions of different turbulence intensity. Annual review of fluid mechanics 46:567-590

Vanderwel C and Ganapathisubramani B (2015) Effects of spanwise spacing on large-scale secondary flows in rough-wall turbulent boundary layers. Journal of Fluid Mechanics 774:1-12

Wangsawijaya DD, Baidya R, Chung D, Marusic I, and Hutchins N (2020) The effect of spanwise wavelength of surface heterogeneity on turbulent secondary flows. Journal of Fluid Mechanics 894:A7

Watanabe T, Zhang X, and Nagata K (2018) Turbulent/non-turbulent interfaces detected in DNS of incompressible turbulent boundary layers. Physics of Fluids 30:035102

Wu D, Wang J, Cui G, and Pan C (2020) Effects of surface shapes on properties of turbulent/non-turbulent interface in turbulent boundary layers. Science China Technological Sciences 63:214-222 